bruise on his occiput. Immediately, he fell into a comatose state continuing for two days. From the 4 th after the accident he became delirious-indistinct state and then irritable in the 30 th.

In the 150 th, he lossed restraint and revealed euphoria, Moria and Korsakow's syndrome.

Neurologically, he revealed hyperrefrexia, spascity and rigidity of limbs, ataxia and urine incontinence. From the 190th, he fell into coma and death occurred on the 204th.

Pathological: The right frontal artery was blocked and atherom-sclerosis was seen in several cerebral major arteries. The orbital part of frontal was destructed and lost. In the vicinity of this focus, new small haemorrhage was observed.

The second case: Male patient of eighty-eight years of age. He also collided against the car into unconsciousness. From the 15th exciting state was observed. After the 70th, he revealed Korsakow's syndrome and loss of restraint. Although he was recovering consciousness at the 80 th, general state changed for the worse to die on the 100th after the accident.

Pathological: Chronic subarachenoideal haematoma covered the brain from left front to occiput. Atherom-sclerosis was observed in the several major arteries such as carotis, basis, middle brain and frontal. In the left frontal, there was laceration of the surface of brain tissue and haemorrhagic necrosis was seen in the bilateral semioval.

\title{
140. Studies on the Pharmacological Therapy for Sequelae of Brain Damage
}

\author{
Shigeo Watanabe and Kazuo Mrwa \\ Department of Neurosurgery, Central General Hospital of Social Insurance, Tokyo
}

It's very difficult to recover or prevention of sequelae of brain damage, therefore, these symptoms almost irreversible to cure, so that shows very miserable in future.

To use effective medicaments for brain-disfunction in acute stadium of brain damage is very important, and so, studied numerous pharmacological therapy until now. (for example, GABA, GABOB, F.A.D. (Flavin-adenine-dinucleotide), Cytochrome $\mathrm{C}$, Actihaemyl, Cerebrolysin, Vitamine $\mathrm{B}_{12}, \mathrm{~B}_{6}, \mathrm{~B}_{2}, \mathrm{~B}_{1}$, Centrophenxine, Cytidine diphosphate cholin, Adenosine triphosphate, Pyridoxal-5-phosphate, etc.)

Above all, among various medicaments chlorophll-a has a strongest effect and with the aid of histochemical techniques, oxidative enzyme activity of the 
cells of the brain was demonstrated to recover the disfunction of damaged-brain.

There are three important derivate of polpyllinsystem, namely, Cytochrome $\mathrm{C}$, Vitamin $\mathrm{B}_{12}$, and chlorophyll-a. (1, 3, 5, 8,-tetramethyl-4-ethyl-2-vinyl-9-oxo-10carbomethoxy-phorbin-7-propionic acid phytylester-magnesium chelate).

The three co-operators accelerate and activate metabolism of nerve-cell, especially, chlorophyll-a gives to energy which occurred to oxydation of glucose by cytochrome to ADP, then changes ATP.

Recently, 52 patients with post-traumatic sequelae (headache, vertigo, paresis, paresthesia, epilepsy, neuralgia, speech disturbance, hearing disturbance and etc.) were examined with the use of chlorophll-a.

The clinical results were as follows: well improved 30\%, improved 50\%, slighlty improved $13 \%$, not changed $2 \%$.

\title{
141. Experience on the Surgical Treatment in Severe Cerebral Contusion
}

\author{
Hiroyasu Makino, Tsuyoshi Hirashima, Akira Yamaura \\ and Tohru TAKeshima \\ 2nd Department of Surgery, Chiba University School of Medicine
}

There is no definite principle in the treatment of severe cerebral contusion. Some people perform decomprssion craniotomy and othrs treat conservatively with hypothermia and heavy steroid therapy. We have observed 12 cases of severe cerebral contusion during the past one year and performed decompression craniotomy in all cases.

Usually a flap was made with a large craniotomy and bone flap was merely placed on the defect without suturing. Otherwise left to be removed from the skull to reduce the intracranial pressure. Fascia of the temporal muscle or the fascia lata was grafted to dural defect, occasionally, expecting better decompressive effects.

In infants, tracheostomy was performed in almost all cases and gastrostomy in those unconscious over a week for the nutritional improvement.

The results were as follows: 6 cases were completely cured, 2 cases improved, 3 died ( 2 of them being in the state of apnoea on arrival), and one case was continuously unconscious over 10 months with tracheostomy and gastrostomy.

We consider that this kind of surgical treatment is often very effective in severe cerebral contusion. 